\title{
Impact of hepatitis $C$ virus genotype- 4 eradication following direct acting antivirals on liver stiffness measurement
}

Mohammed Tag-Adeen ${ }^{1,2}$

Ahlam Mohamed Sabra'

Yuko Akazawa ${ }^{2}$

Ken Ohnita ${ }^{2}$

Kazuhiko Nakao ${ }^{2}$

'Department of Internal Medicine, Qena School of Medicine, South Valley University, Qena, Egypt; 'Department of Gastroenterology and Hepatology, Nagasaki School of Biomedical Sciences, Nagasaki University, Nagasaki, Japan
Correspondence: Mohammed Tag-Adeen Department of Internal Medicine, Qena School of Medicine, South Valley University, Qena 83523, Egypt Fax +2096533757

Email tagmedicine@gmail.com
This article was published in the following Dove Press journal:

Hepatic Medicine: Evidence and Research

6 October 2017

Number of times this article has been viewed

Background: Liver fibrosis is the most important prognostic factor in chronic hepatitis $\mathrm{C}$ virus (HCV) patients, and Egypt shows the highest worldwide HCV prevalence with genotype-4 predominance. The aim of this study was to investigate the degree of liver stiffness measurement (LSM) improvement after successful HCV eradication.

Patients and methods: The study included 84 chronic HCV Egyptian patients, and was conducted at Qena University Hospital from November 1, 2015 till October 31, 2016. LSM was obtained by FibroScan ${ }^{\circledR}$ before starting direct acting antiviral (DAA) treatment and after achieving sustained virologic response-24 (SVR-24). Based on baseline LSM, patients were stratified into F0-F1, F2, F3 and F4 groups (METAVIR). LSM and laboratory data after achieving SVR-24 was compared with that before starting therapy in each fibrosis group (F0-F4), $p$-value $<0.05$ was statistically significant Results: Following DAA treatment, 80 patients achieved SVR-24; of these, 50 were males (62.5\%), mean age: $54.2 \pm 7.6$ years, and mean body mass index: $28.6 \pm 2.2 \mathrm{~kg} / \mathrm{m}^{2}$. Mean baseline LSM dropped from $15.6 \pm 10.8$ to $12.1 \pm 8.7 \mathrm{kPa}$ post-SVR; the maximum change of -5.8 occurred in F4 versus $-2.79,-1.28$ and +0.08 in F3, F2 and F0 -F1 respectively $(p<0.0001)$. At baseline, 41 patients were in the F4 group; only $16(39 \%)$ regressed to non-cirrhotic range $(<12.5 \mathrm{kPa})$, while $25(61 \%)$ were still cirrhotic despite achieving SVR-24 $(p<0.0001)$. Patients who achieved LSM improvement ( $\mathrm{n}=64)$ have had significantly higher baseline aspartate transferase (AST) and alanine transaminase (ALT). Also, those patients showed significant improvement in AST, AST/ platelets ratio index (APRI) and fibrosis-4 index (Fib-4) after achieving SVR; 91\% showed AST improvement $(p=0.01)$ and APRI improvement $(p=0.01)$ and $81 \%$ showed Fib-4 improvement $(p=0.04)$. Females, diabetics, patients with S3 steatosis and patients older than 50 years showed less LSM improvements than their counterparts. Baseline LSM $\geq 9 \mathrm{kPa}$, bilirubin $\geq 1 \mathrm{mg} / \mathrm{dl}$, ALT $\geq 36 \mathrm{U} / \mathrm{L}$ and AST $\geq 31 \mathrm{U} / \mathrm{L}$ were significant predictors for LSM improvement.

Conclusion: Successful HCV genotype-4 eradication results in significant LSM improvement; the best improvement occurs in F4 patients. But as the majority of cirrhotics are still at risk for liver decompensation and hepatocellular carcinoma development despite achieving SVR-24, early detection and treatment are highly recommended.

Keywords: hepatitis $\mathrm{C}$ virus, direct acting antivirals, liver stiffness measurements, FibroScan, transient elastography

\section{Introduction}

Hepatitis C Virus (HCV) is a common health problem in Egypt, and because most of patients are unaware by their illness, many cases are discovered lately after occurrence of substantial liver fibrosis. Recently, direct acting antivirals (DAAs), medicines with direct activity against $\mathrm{HCV}$, were introduced for treatment of $\mathrm{HCV}$ with very favorable 
results regarding viral cure, but liver fibrosis is still an unresolved problem. Our study was done to assess to what degree viral cure results in regression of liver fibrosis. In this study, we measured liver fibrosis by a special device, FibroScan, which measures stiffness of liver in a painless non-invasive manner by putting a small probe on the patient's lower chest. This probe transduces ultrasound waves that travel through the liver to assess its stiffness; the more the liver stiffness, the more the fibrosis. To assess the degree of liver fibrosis, the liver stiffness for each patient was measured before starting DAA treatment, then it was measured again 6 months after the end of treatment, and the two readings were compared. Our results showed that only $39 \%$ of patients with severe fibrosis, cirrhosis, regressed to less severe degree of fibrosis, while the majority, 61\%, are still cirrhotics and still at risk for developing liver dysfunction and liver cancer. Based on this result, addition of a screening program to the national project for control of HCV in Egypt that enables early detection and treatment of HCV patients is highly recommended.

HCV affects approximately 130-150 million people worldwide. The majority of affected individuals develop a chronic infection, and approximately $30 \%$ of them will progress to cirrhosis within 20-30 years following infection. Therefore, $\mathrm{HCV}$ is a leading cause of end-stage liver disease and hepatocellular carcinoma (HCC). ${ }^{1-4}$ However, it has been proven that effective antiviral treatment modifies the natural history of chronic HCV, reduces fibrosis and decreases the subsequent HCV-related complications and mortality. ${ }^{1,5-7}$

Sustained virologic response-24 (SVR-24), undetected HCV RNA by sensitive amplification technique 24 weeks after the end of treatment, has traditionally been considered the aim of HCV treatment and the virologic cure of $\mathrm{HCV}$ infection. ${ }^{8,9}$ The current DAAs have remarkably increased SVR rates to $88 \%-90 \%$, while the newer DAA regimens likely to be approved later will effectively treat genotype-3, now the difficult-to-treat genotype, and this will bring SVR rates across all genotypes to $98 \%{ }^{10-12}$

In Egypt, the prevalence of HCV infection is the highest in the world, ${ }^{13,14}$ and genotype-4 is the most prevalent genotype. ${ }^{15-17}$ Although screening for $\mathrm{HCV}$ is recommended ${ }^{18,19}$ and the current available screening test has a $97 \%$ sensitivity and $100 \%$ specificity, ${ }^{20-22}$ up to $60 \%$ of cases in Egypt are diagnosed with decompensated cirrhosis or $\mathrm{HCC}^{23,24}$ mainly due to the lack of a national screening program.

Transient elastography (TE) is a novel, non-invasive, ultrasound technique-based technology that assess liver stiffness measurement (LSM). Established evidences have indicated that LSM has good sensitivity and specificity for liver fibrosis and cirrhosis. So, it has become popular over the past few years especially for obtaining repeated measurements because it eliminates pain, morbidity and mortality that may accompany liver biopsy. ${ }^{25-27}$

This study was conducted to investigate degree of LSM improvement after successful HCV eradication following DAA treatment in Egyptian patients.

\section{Patients and methods}

This study was conducted in Qena University Hospital from November 1, 2015 till October 31, 2016 among 84 Egyptian patients with chronic HCV diagnosed by PCR HCV RNA. All patients were subjected to TE before starting DAA treatment and after achieving SVR-24, with recording of clinical and laboratory data at baseline and after achieving SVR-24. Based on the baseline LSM, patients were stratified according to estimated METAVIR fibrosis score into F0-F1, F2, F3 and F4 groups.

\section{Ethical clearance}

The study protocol was approved by the Ethical Committee of Faculty of Medicine, South Valley University, Qena, Egypt. A written informed consent was obtained from each patient enrolled in the study.

\section{Transient elastography}

TE and controlled attenuation parameter (CAP) were obtained using FibroScan $\AA$ device (FibroScan, Echosens, Paris, France) by an expert FibroScan operator, who was blinded about patient's data and the outcome of DAA treatment.

- LSM was performed after 8 hours of fasting in the right lobe of the liver through the intercostal spaces with the patient in the supine position, and it was expressed in $\mathrm{kPa}$.

- Result would not be considered reliable except after acquisition of 12 successful readings, with interquartile range/median ratio less than $30 \%$.

- LSM was used to estimate the METAVIR fibrosis stage as follows: F0-F1: 2.5-6.9 kPa; F2: 7.0-9.4 kPa; F3: $9.5-12.4 \mathrm{kPa} ; \mathrm{F} 4: \geq 12.5 \mathrm{kPa}{ }^{28}$

- CAP was expressed in $\mathrm{dB} / \mathrm{m}$ and its values were used to estimate steatosis stage as follows: $\mathrm{S} 0<238 \mathrm{~dB} / \mathrm{m}, \mathrm{S} 1$ : $238-258 \mathrm{~dB} / \mathrm{m}, \mathrm{S} 2: 259-291 \mathrm{~dB} / \mathrm{m}$ and S3: $\geq 292 \mathrm{~dB} / \mathrm{m}^{29}$

\section{DAA treatment}

Different combinations of DAA were used according to the functional state of the patient, including sofosbuvir/daclatasvir for 12 weeks in 35 patients, sofosbuvir/daclatasvir for 24 weeks in 15 patients, sofosbuvir/daclatasvir/ribavirin for 12 weeks in 15 patients, sofosbuvir/ribavirin for 24 weeks in 
11 patients, sofosbuvir/ledipasvir for 12 weeks in 3 patients and sofosbuvir/ledipasvir for 24 weeks in 1 patient. SVR-24 was defined as sustained loss of HCV RNA at 24 weeks after the end of treatment.

\section{Exclusion criteria}

- Patients with baseline body mass index (BMI) $>35 \mathrm{~kg} / \mathrm{m}^{2}$

- Patients with ascites, HCC or any hepatic focal lesion

- Patients with combined HCV/hepatitis B virus (HBV) infection and/or bilharziasis

- Alcoholics and/or intravenous drug abusers

\section{Statistical analysis}

Continuous variables were expressed as mean and standard deviation. Categorical variables were presented as absolute numbers and percentages. Data were analyzed using two-tailed paired $t$-test, pooled $t$-test, analysis of variance, Fisher's exact test and nominal regression. A $p$-value $<0.05$ was considered statistically significant. All statistical analyses were performed with JMP ${ }^{\circledR}$ version 11, SAS Institute Inc., Cary, NC, USA.

\section{Results}

A total of 84 chronic HCV Egyptian patients were included at the start of this study but 4 were excluded because they did not achieve SVR-24. Table 1 shows baseline clinical and laboratory data of the study population. According to esti-

Table I Baseline clinical and laboratory data

\begin{tabular}{|c|c|}
\hline $\begin{array}{l}\text { Baseline clinical and laboratory data } \\
(n=80)\end{array}$ & \\
\hline Age (years) & $54.2 \pm 7.6(36-77)$ \\
\hline Body mass index $\left(\mathrm{kg} / \mathrm{m}^{2}\right)$ & $28.6 \pm 2(24-34.6)$ \\
\hline Sex $(\text { male })^{\mathrm{a}}$ & $50(62.5 \%)$ \\
\hline Diabetics $^{\mathrm{a}}$ & $12(15 \%)$ \\
\hline Alanine transaminase $(\mathrm{U} / \mathrm{L})$ & $6 I \pm 4 I(12-199)$ \\
\hline Aspartate transaminase $(\mathrm{U} / \mathrm{L})$ & $63 \pm 4 I(6-202)$ \\
\hline Serum albumin (g/dL) & $3.6 \pm 0.5(2.1-5)$ \\
\hline Serum bilirubin (mg/dL) & $0.8 \pm 0.5(0.1-3)$ \\
\hline International normalization ratio & $I . I \pm 0.1(0.9-1.9)$ \\
\hline Platelets $\left(\times 10^{3}\right)$ & $174.9 \pm 82(60-378)$ \\
\hline Hemoglobin (g/dL) & $12.5 \pm 1.6(9.2-18)$ \\
\hline Leucocytic count $\left(\times 10^{3}\right)(\mathrm{WBCs})$ & $6.5 \pm 2.4(2.2-16)$ \\
\hline Fasting blood glucose (mg/dL) & $132 \pm 80(67-390)$ \\
\hline Creatinine (mg/dL) & $0.7 \pm 0.3(0.1-1.8)$ \\
\hline Aspartate transferase/platelets ratio index & $1.3 \pm 1.4$ \\
\hline Fibrosis-4 index & $3.3 \pm 2.6$ \\
\hline Liver stiffness measurement $(\mathrm{kPa})$ & $15.6 \pm 1 \mid(3-51.9)$ \\
\hline Controlled attenuation parameter $(\mathrm{dB} / \mathrm{m})$ & $231.7 \pm 46(100-357)$ \\
\hline
\end{tabular}

Note: Data presented as mean \pm standard deviation (range), or ${ }^{\mathrm{a}}$ number and percent. mated METAVIR score based on baseline LSM, 18 patients $(23 \%)$ were in $\mathrm{F} 0-\mathrm{F} 1,12(15 \%)$ in $\mathrm{F} 2,9(11 \%)$ in $\mathrm{F} 3$ and $41(51 \%)$ in $\mathrm{F} 4$ fibrosis groups. Regarding previous antiHCV treatment, 2 (2.5\%) patients were sofosbuvir+ribavirin experienced, 3 (3.7\%) were PEGylated interferon+ribavirin experienced and the remaining 75 (93.8\%) were naive.

Table 2 shows that achievement of SVR-24 was associated with statistically significant reduction of alanine transaminase (ALT), aspartate transaminase (AST), serum bilirubin, international normalization ratio (INR), hemoglobin, fasting blood glucose (FBG), AST/platelets ratio index (APRI), fibrosis-4 index (Fib-4) and LSM, and statistically significant increase in BMI, albumin and creatinine, while both platelets and CAP showed insignificant increase. Comparison among variables across different fibrosis groups is shown in Table 3, where all groups have shown improvement in ALT, AST, APRI, Fib-4, bilirubin, INR and albumin levels. LSM improvement occurred in patients with significant fibrosis (F2-F4 groups), with the maximum improvement in F4 $(-5.8)$ versus F3, F2 and F0-F1 $(-2.8,-1.3$ and $+0.08 \mathrm{kpa}$, respectively), $p$-value $<0.0001$; Figure 1 .

Mean percent change of LSM was significantly better in cirrhotics $(-25 \%)$ versus non-cirrhotics $(-8 \%), p=0.004$; Figure 2. But regarding age, sex, diabetes and steatosis, the differences were not statistically significant (Figure 3 and Table 4).

At baseline, there were 41 cirrhotics; however, after achieving SVR, only 16 of them (39\%) regressed to subcirrhotic range $(<12.5 \mathrm{kPa})$. Cirrhosis regressed to $\mathrm{F} 3$ in $11, \mathrm{~F} 2$ in 3 and F0-F1 in 2 patients, while the majority (25 patients [61\%]) remained as F4. In all patients after achieving SVR, fibrosis regressed in 31 (38.7\%), remained stable in $46(57.5 \%)$ and paradoxically worsened in $3(3.7 \%)$ (Table 5).

Patients who did achieve LSM improvement ( $\mathrm{n}=64)$ have had significantly higher LSM, bilirubin, ALT and AST at baseline in comparison with patients who did not $(n=16)$, while differences in age, albumin, INR and platelets were not significant (Table 6). Also, in those patients $(n=64)$, LSM improvement was significantly associated with AST and APRI improvements in 91\% $(p=0.01)$ and with Fib-4 improvement in $81 \%(p=0.04)$, while the association of LSM improvement was insignificant with ALT reduction $(p=0.9)$ and platelet improvement $(p=0.06)$.

Baseline LSM $\geq 9 \mathrm{kPa}$, bilirubin $\geq 1 \mathrm{mg} / \mathrm{dL}, \mathrm{ALT} \geq 36 \mathrm{U} / \mathrm{L}$ and $A S T \geq 31 \mathrm{U} / \mathrm{L}$ were statistically significant predictors for achieving LSM improvement after SVR-24. 
Table 2 Comparison among variables at baseline and after achieving SVR (post-SVR)

\begin{tabular}{|c|c|c|c|c|}
\hline Variables $(n=80)$ & Baseline & Post-SVR & Mean change & $p$-value \\
\hline BMI $\left(\mathrm{kg} / \mathrm{m}^{2}\right)$ & $28.60 \pm 2.2(24-34.6)$ & $28.69 \pm 2.2(24-35)$ & $+0.08(-0.6-2)$ & $0.02 *$ \\
\hline ALT (U/L) & $6 I \pm 4 \mid(12-199)$ & $24.8 \pm 14.9(4-118)$ & $-36(-152-14)$ & $<0.000 I^{*}$ \\
\hline AST (U/L) & $63 \pm 4 \mid(6-202)$ & $27.3 \pm 13.1(7-80)$ & $-35.8(-160-18)$ & $<0.000 I^{*}$ \\
\hline Albumin (g/dL) & $3.6 \pm 0.5(2.1-5)$ & $3.9 \pm 0.5(3-5.2)$ & $+0.28(-0.6-1.4)$ & $<0.000 I^{*}$ \\
\hline Bilirubin (mg/dL) & $0.8 \pm 0.5(0.1-3)$ & $0.7 \pm 0.3(0.1-2.1)$ & $-0.14(-1.4-0.8)$ & $<0.000 I^{*}$ \\
\hline INR & $1.1 \pm 0.1(0.9-1.9)$ & I.04₫0.I (0.3-I.7) & $-0.08(-0.6-0.2)$ & $<0.000 I^{*}$ \\
\hline Platelets $\left(\times 10^{3}\right)$ & $174.9 \pm 82(60-378)$ & $\mid 86.4 \pm 78.9(5|-4| \mid)$ & $+11.5(-154-218)$ & 0.08 \\
\hline Hemoglobin (g/dL) & $12.5 \pm 1.6(9.2-18)$ & $12.1 \pm 1.7(8.7-17.8)$ & $-0.39(-3-4.4)$ & $0.004^{*}$ \\
\hline WBCs $\left(\times 10^{3}\right)$ & $6.5 \pm 2.4(2.2-16)$ & $6.2 \pm 2.3(2.5-14)$ & $-0.31(-5.5-8)$ & 0.2 \\
\hline FBG (mg/dL) & $132 \pm 80(67-390)$ & $107.7 \pm 47.3(69-320)$ & $-24.4(-300-40)$ & $0.0001 *$ \\
\hline Creatinine (mg/dL) & $0.7 \pm 0.3(0.1-1.8)$ & $0.8 \pm 0.3(0.1-1.7)$ & $+0.07(-0.4-1)$ & $0.009 *$ \\
\hline APRI & $1.3 \pm 1.4$ & $0.5 \pm 0.3$ & -0.8 & $<0.000 I^{*}$ \\
\hline Fib-4 & $3.3 \pm 2.6$ & $2.1 \pm 1.5$ & -1.2 & $<0.000 I^{*}$ \\
\hline $\operatorname{LSM}(\mathrm{kPa})$ & $|5.6 \pm| \mid(3-5 \mid .9)$ & $12.1 \pm 8.7(3.5-45.7)$ & $-3.5(-21.9-5.8)$ & $<0.000 I^{*}$ \\
\hline $\mathrm{CAP}(\mathrm{dB} / \mathrm{m})$ & $231.7 \pm 46(100-357)$ & $234.5 \pm 51.2(100-354)$ & $+2.8(-259-121)$ & 0.5 \\
\hline
\end{tabular}

Notes: Data presented as mean \pm standard deviation (range), paired $t$-test. *Statistically significant values.

Abbreviations: ALT, alanine transaminase; APRI, AST/platelets ratio index; AST, aspartate transaminase; BMI, body mass index; CAP, controlled attenuation parameter; FBG, fasting blood glucose; Fib-4, fibrosis-4 index; INR, international normalization ratio; LSM, liver stiffness measurement; SVR, sustained virologic response; WBC, white blood cells.

\section{Discussion}

It has been proven that HCV eradication and achieving SVR result in improved mortality and decreased risk in HCV-related complications. Although the mechanism underlying this decrease in-risk is yet to be fully determined, there is agreement that fibrosis regression plays a pivotal role. ${ }^{1}$ SVR has been considered the goal of $\mathrm{HCV}$ treatment; however, liver fibrosis rather than serum viremia is the most important prognostic factor in chronic $\mathrm{HCV}$ patients. $^{30}$

For our knowledge, most of published articles studied fibrosis changes in $\mathrm{HCV}$ patients following interferon therapy and were not limited to certain HCV genotype, while this study was limited to genotype-4 patients treated with DAAs. Also, this study is the first to compare LSM improvement in patients aged $\leq 50$ vs $>50$ years, diabetics vs non-diabetics and $\mathrm{S} 0-\mathrm{S} 2$ vs S3 steatosis.

Because LSM is disease specific, ${ }^{31}$ only chronic HCV patients were included, while those with other liver diseases such as chronic HBV, HCC, bilharziasis and alcoholics were excluded. Intravenous drug users were also excluded to avoid inclusion of HCV genotypes other than genotype-4. ${ }^{15}$

Our results showed that achieving SVR-24 was associated with statistically significant overall change of LSM by a mean of $-3.5 \mathrm{kPa}$ (range: -21.9 to +5.8 ), which is consistent with many other studies ${ }^{7,32-35}$ (Figure 4). However, the used anti-HCV treatment in our study was DAA, while it was interferon or interferon and DAA in the others.
The best improvement in LSM occurred in F4 patients with mean percent of $-25 \%$ versus $-8 \%$ in $\mathrm{F} 0-\mathrm{F} 3, p$-value 0.004 . In the study by Sweta et al, ${ }^{32}$ median percent changes were $-44 \%$ in cirrhotics versus $-20 \%$ in non-cirrhotics.

Despite the fact that it was accompanied by significant improvement in LSM, Fib-4, APRI and liver biochemistry, achieving SVR did not guarantee improvement of cirrhosis; only $39 \%$ of cirrhotics regressed to sub-cirrhotic range (LSM $<12.5 \mathrm{kPa}$ ); relatively near results were noticed by previous studies. ${ }^{32,36-40}$ So, most of cirrhotics $(61 \%)$ are still having risk for liver decompensation and HCC development despite achieving SVR-24. This finding necessitates early treatment before occurrence of permanent liver damage to get the ultimate goal of HCV eradication.

S3 steatosis, diabetes, female sex and age above 50 years have worse outcome regarding LSM improvement, and it is unclear that treating steatosis and tight glycemic control in adjunction with DAA might help in better result or not.

Baseline ALT and AST were higher in patients who did achieve LSM improvement ( $n=64)$ than in patients who did not $(n=16)$ (Figure 5). However, LSM improvement showed statistically significant association with $\operatorname{AST}(p=0.01)$ rather than with ALT $(p=0.9)$; this important notice may explain that LSM improvement following SVR might be due to regression of fibrosis rather than resolution of necroinflammatory activity.

The main limitation of our study was the lack of histological evidence that confirms if LSM improvement could 
Table 3 Comparison among variables at baseline and post-SVR across different fibrosis groups

\begin{tabular}{|c|c|c|c|c|c|c|}
\hline Variables & & F0-FI $(n=\mid 8)$ & $F 2(n=12)$ & F3 $(n=9)$ & $F 4(n=4 I)$ & p-value \\
\hline$\overline{\mathrm{ALT}}$ & Baseline & $51 \pm 37$ & $62 \pm 44$ & $57 \pm 37$ & $66 \pm 42$ & 0.6 \\
\hline \multirow[t]{2}{*}{$(\mathrm{U} / \mathrm{l})$} & Post-SVR & $19 \pm 7.2$ & $26 \pm 12$ & $15 \pm 5$ & $29 \pm 18$ & $0.01 *$ \\
\hline & Change & -32 & -36 & -42 & -37 & 0.9 \\
\hline AST & Baseline & $48 \pm 29$ & $57 \pm 41$ & $53 \pm 28$ & $74 \pm 45$ & 0.1 \\
\hline \multirow[t]{2}{*}{ (U/L) } & Post-SVR & $22 \pm 9$ & $27 \pm 11$ & $20 \pm 4$ & $31 \pm 15$ & $0.01 *$ \\
\hline & Change & -26 & -30 & -33 & -43 & 0.4 \\
\hline Albumin & Baseline & $4.03 \pm 0.4$ & $3.9 \pm 0.5$ & $3.8 \pm 0.4$ & $3.3 \pm 0.5$ & $<0.0001^{*}$ \\
\hline \multirow[t]{2}{*}{$(g / d L)$} & Post-SVR & $4.3 \pm 0.4$ & $4.18 \pm 0.5$ & $4.2 \pm 0.4$ & $3.6 \pm 0.4$ & $<0.000 I^{*}$ \\
\hline & Change & +0.27 & +0.28 & +0.4 & +0.3 & 0.8 \\
\hline Bilirubin & Baseline & $0.58 \pm 0.18$ & $0.6 \pm 0.25$ & $0.7 \pm 0.4 \mathrm{I}$ & $1.16 \pm 0.53$ & $<0.000 I^{*}$ \\
\hline \multirow[t]{2}{*}{$(\mathrm{mg} / \mathrm{dL})$} & Post-SVR & $0.51 \pm 0.19$ & $0.5 \pm 0.18$ & $0.68 \pm 0.31$ & $0.94 \pm 0.4 \mathrm{I}$ & $<0.000 I^{*}$ \\
\hline & Change & -0.07 & -0.1 & -0.02 & -0.22 & 0.2 \\
\hline \multirow[t]{3}{*}{ INR } & Baseline & $1.02 \pm 0.06$ & $1.03 \pm 0.09$ & $1.08 \pm 0.1$ & $1.2 \pm 0.21$ & $0.0001 *$ \\
\hline & Post-SVR & $0.95 \pm 0.17$ & $0.99 \pm 0.05$ & $1.03 \pm 0.12$ & $1.10 \pm 0.16$ & $0.003^{*}$ \\
\hline & Change & -0.07 & -0.04 & -0.05 & -0.1 & 0.3 \\
\hline Hemoglobin & Baseline & $13.1 \pm 2$ & $13.5 \pm 1.38$ & $12.9 \pm 1.39$ & $11.9 \pm 1.43$ & $0.007^{*}$ \\
\hline \multirow[t]{2}{*}{$(\mathrm{g} / \mathrm{dL})$} & Post-SVR & $13 \pm 1.9$ & $13.03 \pm 1.6$ & $12.8 \pm 1.6$ & $1 \mathrm{I} .42 \pm \mathrm{I} .4$ & $0.0007^{*}$ \\
\hline & Change & -0.1 & -0.47 & -0.1 & -0.48 & 0.5 \\
\hline Platelets & Baseline & $267.83 \pm 54$ & $195.6 \pm 44$ & $190.6 \pm 92$ & $124.6 \pm 56$ & $<0.000 I^{*}$ \\
\hline \multirow[t]{2}{*}{$\left(\times 10^{3}\right)$} & Post-SVR & $266.88 \pm 60$ & $212.66 \pm 60$ & $196.6 \pm 76$ & $14 \mid .2 \pm 58$ & $<0.000 I^{*}$ \\
\hline & Change & -0.95 & +17 & +6 & +16.6 & 0.7 \\
\hline \multirow[t]{3}{*}{ APRI } & Baseline & $0.5 \pm 0.3$ & $0.8 \pm 0.7$ & $0.9 \pm 0.6$ & $2 \pm 1.5$ & $0.0002 *$ \\
\hline & Post-SVR & $0.2 \pm 0.09$ & $0.4 \pm 0.2$ & $0.3 \pm 0.2$ & $0.7 \pm 0.4$ & $<0.000 I^{*}$ \\
\hline & Change & -0.3 & -0.4 & -0.6 & -1.3 & $0.009 *$ \\
\hline \multirow[t]{3}{*}{ Fib-4 } & Baseline & $1.4 \pm 0.5$ & $2 \pm 1.3$ & $2.6 \pm 1.5$ & $5 \pm 2.8$ & $<0.000 I^{*}$ \\
\hline & Post-SVR & $\mathrm{I} \pm 0.5$ & $1.5 \pm 0.9$ & $1.7 \pm 0.7$ & $2.7 \pm 1.7$ & $<0.0001 *$ \\
\hline & Change & -0.4 & -0.5 & -0.9 & -2.3 & $0.008 *$ \\
\hline LSM & Baseline & $4.85 \pm 0.9$ & $8.27 \pm 0.9$ & $10.91 \pm 0.8$ & $23.59 \pm 9.7$ & $<0.000 I^{*}$ \\
\hline \multirow[t]{2}{*}{$(\mathrm{kPa})$} & Post-SVR & $4.93 \pm 1.3$ & $6.99 \pm 3$ & $8.12 \pm 1.7$ & $17.79 \pm 8.9$ & $<0.000 I^{*}$ \\
\hline & Change & +0.08 & -1.28 & -2.79 & -5.8 & $<0.000 I^{*}$ \\
\hline CAP & Baseline & $228.5 \pm 39.2$ & $225.9 \pm 55.2$ & $251.6 \pm 45.6$ & $230.5 \pm 47.3$ & 0.5 \\
\hline \multirow[t]{2}{*}{$(\mathrm{dB} / \mathrm{m})$} & Post-SVR & $239.05 \pm 55$ & $228.9 \pm 42$ & $242.8 \pm 40$ & $232.34 \pm 55$ & 0.9 \\
\hline & Change & +10.5 & +3 & -8.8 & +1.8 & 0.4 \\
\hline
\end{tabular}

Notes: Data presented as mean \pm standard deviation, ANOVA. *Statistically significant values.

Abbreviations: ALT, alanine transaminase; APRI, AST/platelets ratio; AST, aspartate transaminase; CAP, controlled attenuation parameter; Fib-4, fibrosis-4 index; index; INR, international normalization ratio; LSM, liver stiffness measurement; SVR, sustained virologic response.

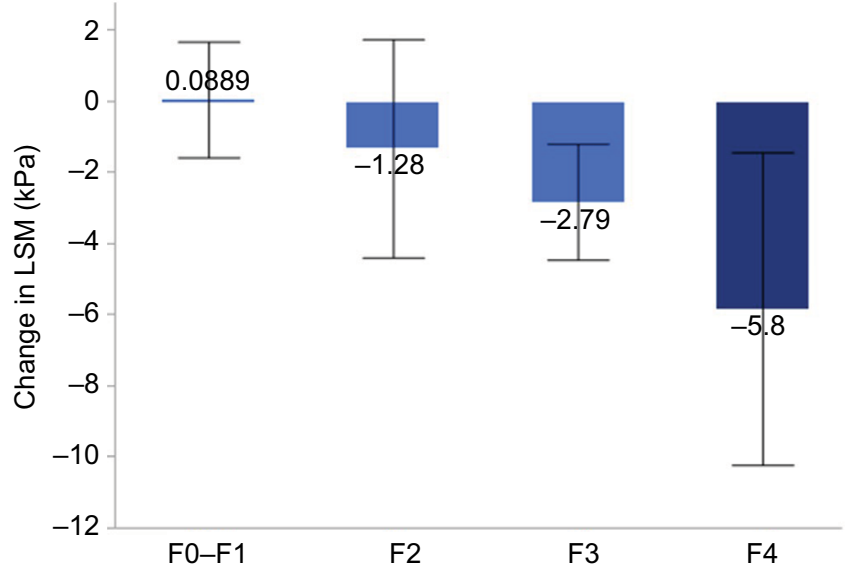

Figure I Mean change in LSM after achieving SVR in different fibrosis groups.

Note: The mean LSM reduction in cirrhotics (F4 group, dark blue column) versus in non-cirrhotics (F0-FI, F2, F3 groups, light blue columns), error bars refer to I $S D$ from the mean.

Abbreviations: LSM, liver stiffness measurement; SVR, sustained virologic response.

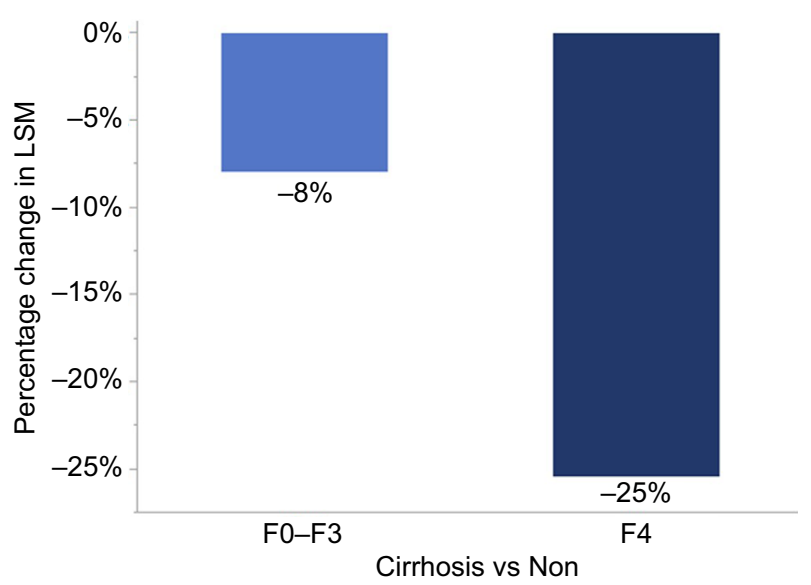

Figure 2 Mean percent change in LSM in cirrhotics versus non-cirrhotics.

Note: Mean percent LSM reduction in cirrhotics (F4 group, dark blue column) versus in non-cirrhotics (F0-F3 group, light blue column).

Abbreviation: LSM, liver stiffness measurement. 

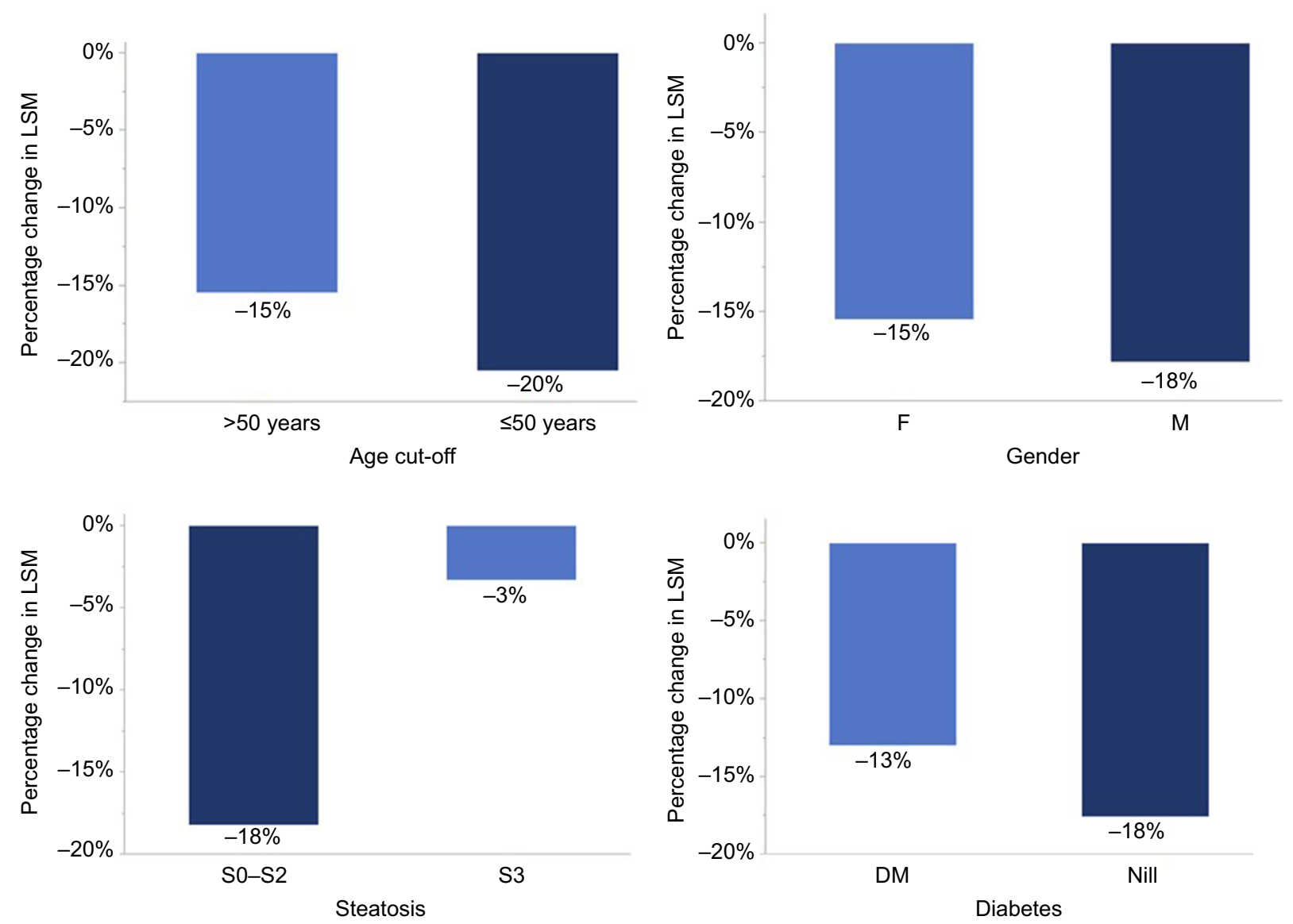

Figure 3 Mean percent change in LSM in different patients' groups regarding age, sex, steatosis and diabetes.

Note: LSM improvement was better in: patients $\leq 50$ years, males, S0-S2 steatosis, and non-diabetics (dark blue columns) than that in: patients older than 50 years, females, S3 steatisis, and diabetics (light blue columns).

Abbreviations: DM, diabetes mellitus; F, female; LSM, liver stiffness measurement; M, male.

Table 4 Mean percent decrease of LSM in different patients' groups

\begin{tabular}{|c|c|c|c|}
\hline \multicolumn{2}{|c|}{ Variables and patient groups } & \multirow{2}{*}{$\begin{array}{l}\text { Mean percent } \\
\text { LSM change (\%) } \\
-20 \%\end{array}$} & \multirow{2}{*}{$\begin{array}{l}p \text {-value } \\
0.4\end{array}$} \\
\hline Age & $\leq 50$ & & \\
\hline \multirow[t]{3}{*}{ (years) } & $(n=24)$ & & \\
\hline & $>50$ & $-15 \%$ & \\
\hline & $(n=56)$ & & \\
\hline \multirow[t]{4}{*}{ Sex } & Male & $-18 \%$ & 0.7 \\
\hline & $(n=50)$ & & \\
\hline & Female & $-15.4 \%$ & \\
\hline & $(n=30)$ & & \\
\hline \multirow[t]{4}{*}{ Diabetes } & Non-diabetic & $-17 \%$ & 0.6 \\
\hline & $(n=68)$ & & \\
\hline & Diabetic & $-13 \%$ & \\
\hline & $(n=12)$ & & \\
\hline \multirow[t]{4}{*}{ Steatosis } & SO-S2 & $-18 \%$ & 0.1 \\
\hline & $(n=73)$ & & \\
\hline & S3 & $-3 \%$ & \\
\hline & $(n=7)$ & & \\
\hline \multirow[t]{4}{*}{ Cirrhosis } & Cirrhotics (F4) & $-25 \%$ & $0.004 *$ \\
\hline & $(n=4 I)$ & & \\
\hline & Non-cirrhotics (F0-F3) & $-8 \%$ & \\
\hline & $(n=39)$ & & \\
\hline
\end{tabular}

Note: *Statistically significant value.

Abbreviation: LSM, liver stiffness measurement.

Table 5 Estimated fibrosis based on baseline LSM versus estimated fibrosis based on post-SVR LSM

\begin{tabular}{lllllll}
\hline \multicolumn{5}{c}{} & \multicolumn{3}{l}{ Estimated fibrosis based on } & Total \\
\cline { 3 - 6 } & & Fost-SVR LSM & & \\
\cline { 2 - 6 } & & F0-FI & F2 & F3 & F4 & \\
Estimated & F0-FI & I7 (94\%) & I (6\%) & $0(0 \%)$ & $0(0 \%)$ & 18 \\
fibrosis & F2 & $8(67 \%)$ & $2(17 \%)$ & I (8\%) & I (8\%) & 12 \\
based on & F3 & $2(22 \%)$ & $5(56 \%)$ & $2(22 \%)$ & $0(0 \%)$ & 9 \\
baseline & F4 & $2(5 \%)$ & $3(7 \%)$ & II (27\%) & 25 & 4 I \\
LSM & & & & & $(61 \%)$ & \\
Total & & 29 & II & I4 & 26 & 80 \\
\hline
\end{tabular}

Note: Fischer's exact test, $p$-value $<0.0001$.

Abbreviations: LSM, liver stiffness measurement; SVR, sustained virologic response.

predominantly be related to the regression of fibrosis or resolution of necroinflammatory activity. Undoubtedly, invasiveness of liver biopsy with possibility of complications and disagreement of patients were main obstacles in doing biopsy. Also, US-based TE techniques are generally limited by the distance of liver from skin, high technical failure rates and 
Table 6 Comparison between baseline variables in patients who did achieve improvement of LSM versus patients who did not

\begin{tabular}{llll}
\hline Variables & $\begin{array}{l}\text { LSM } \\
\text { improvement } \\
(\mathbf{n}=64)\end{array}$ & $\begin{array}{l}\text { No LSM } \\
\text { improvement } \\
(\mathbf{n}=16)\end{array}$ & p-value \\
\hline LSM $(\mathrm{kPa})$ & $17.1 \pm \mathrm{II}$ & $9.6 \pm 8$ & $0.01^{*}$ \\
Bilirubin (mg/dL) & $0.9 \pm 0.54$ & $0.6 \pm 0.18$ & $0.03^{*}$ \\
AST (U/L) & $68 \pm 40$ & $44 \pm 39$ & $0.04^{*}$ \\
ALT (U/L) & $66 \pm 4 \mathrm{I}$ & $43 \pm 3 \mathrm{I}$ & $0.04^{*}$ \\
Platelets $\left(\times 10^{3}\right)$ & $166 \pm 80$ & $209 \pm 83$ & 0.06 \\
Albumin (g/dL) & $3.5 \pm 0.5$ & $3.8 \pm 0.5$ & 0.08 \\
INR & $1.1 \pm 0.2$ & $\mathrm{I} \pm 0.1$ & 0.1 \\
Age (years) & $54.2 \pm 7.6$ & $54.1 \pm 8$ & 0.9 \\
\hline
\end{tabular}

Notes: Data presented as mean \pm standard deviation, pooled $t$-test. *Statistically significant values.

Abbreviations: ALT, alanine transaminase; AST, aspartate transaminase; INR, international normalization ratio; LSM, liver stiffness measurement. confounding factors including obesity and hepatic inflammation; ${ }^{41-43}$ however, magnetic resonance elastography has a higher technical success and accuracy than these techniques, and it is now available in many leading institutions around the world, with growing clinical experiences and emerging new applications. ${ }^{44-46}$ In conclusion, successful HCV genotype-4 eradication results in significant LSM improvement; the best improvement occurs in F4 patients. But as the majority of cirrhotics are still having risk for liver decompensation and HCC development despite achieving SVR-24, early detection and treatment are highly recommended.

\section{Acknowledgment}

The authors would like to appreciate all patients who contributed to this study.
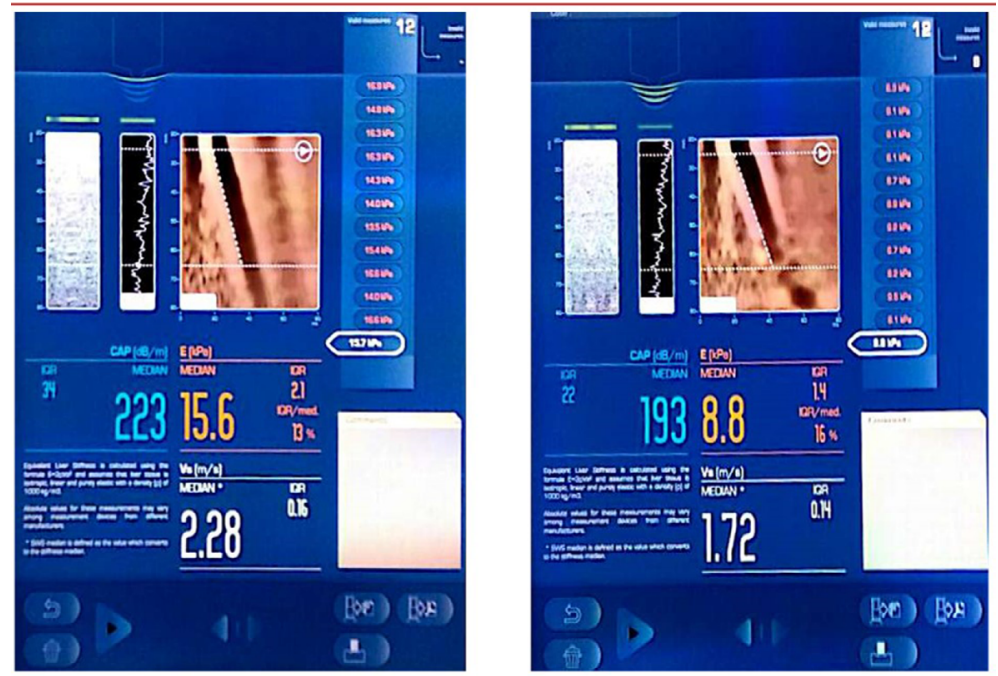

Figure 4 Example of LSM and steatosis improvement after achieving SVR-24.

Abbreviations: LSM, liver stiffness measurement; SVR, sustained virologic response.
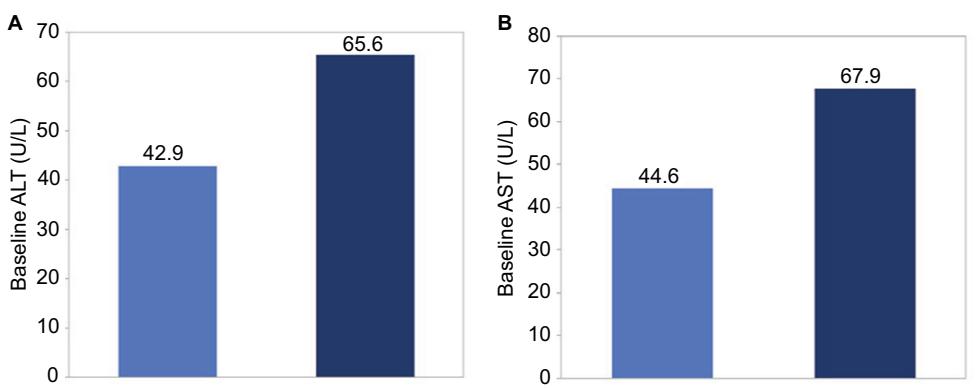

Figure 5 Mean ALT and AST at baseline in patients who did achieve LSM improvement ( $n=64)$ versus patients who did not ( $n=16)$.

Note: Higher baseline ALT (A) and AST (B) are noticed in patients who did achieve LSM improvement (dark blue columns) than in patients who did not (light blue columns). Abbreviations: ALT, alanine transaminase; AST, aspartate transaminase; LSM, liver stiffness measurement. 


\section{Disclosure}

The authors report no conflicts of interest in this work.

\section{References}

1. Gonzalez HC, Duarte-Rojo A. Virologic cure of hepatitis C: impact on hepatic fibrosis and patient outcomes. Curr Gastroenterol Rep. 2016;18(7):32.

2. Perz JF, Armstrong GL, Farrington LA, Hutin YJ, Bell BP. The contributions of hepatitis $\mathrm{B}$ virus and hepatitis $\mathrm{C}$ virus infections to cirrhosis and primary liver cancer worldwide. J Hepatol. 2006;45(4):529-538.

3. Davila JA, Morgan RO, Shaib Y, McGlynn KA, El-Serag HB. Hepatitis $\mathrm{C}$ infection and the increasing incidence of hepatocellular carcinoma: a population-based study. Gastroenterology. 2004;127(5):1372-1380.

4. Poynard T, Bedossa P, Opolon P. Natural history of liver fibrosis progression in patients with chronic hepatitis C. The OBSVIRC, METAVIR, CLINIVIR, and DOSVIRC groups. Lancet. 1997;349(9055):825-832.

5. D'Ambrosio R, Della Corte C, Colombo M. Hepatocellular carcinoma in patients with a sustained response to anti-hepatitis $\mathrm{C}$ therapy. Int $J$ Mol Sci. 2015;16(8):19698-19712.

6. D'Ambrosio R, Aghemo A, Fraquelli M, et al. The diagnostic accuracy of Fibroscan for cirrhosis is influenced by liver morphometry in HCV patients with a sustained virological response. J Hepatol. 2013;59(2): 251-256.

7. Arima $\mathrm{Y}$, Kawabe N, Hashimoto $\mathrm{S}$, et al. Reduction of liver stiffness by interferon treatment in the patients with chronic hepatitis C. Hepatol Res. 2010;40(4):383-392.

8. Yoshida EM, Sulkowski MS, Gane EJ, et al. Concordance of sustained virological response 4,12 , and 24 weeks post-treatment with sofosbuvircontaining regimens for hepatitis C virus. Hepatology. 2015;61(1): $41-45$.

9. Thorlund K, Druyts E, Mills EJ. SVR12 is higher than SVR24 in treatment-naive hepatitis $\mathrm{C}$ genotype 1 patients treated with peginterferon plus ribavirin. Clin Epidemiol. 2014;6:49-58.

10. Foster GR, Afdhal N, Roberts SK, et al; ASTRAL-2 Investigators; ASTRAL-3 Investigators. Sofosbuvir and velpatasvir for HCV genotype 2 and 3 infection. N Engl J Med. 2015;373(27):2608-2617.

11. Lawitz E, Mangia A, Wyles D, et al. Sofosbuvir for previously untreated chronic hepatitis C infection. N Engl J Med. 2013;368(20):1878-1887.

12. Barritt AS 4th, Fried MW. Maximizing opportunities and avoiding mistakes in triple therapy for hepatitis C virus. Gastroenterology. 2012;142(6): $1314 \mathrm{e} 1-1323 \mathrm{e} 1$.

13. Blach S, Zeuzem S, Manns M, et al. Global prevalence and genotype distribution of hepatitis $C$ virus infection in 2015: a modelling study. Lancet Gastroenterol Hepatol. 2017;2(3):161-176.

14. Gomaa A, Allam N, Elsharkawy A, El Kassas M, Waked I. Hepatitis $\mathrm{C}$ infection in Egypt: prevalence, impact and management strategies. Hepat Med. 2017;9:17-25.

15. Ray SC, Arthur RR, Carella A, Bukh J, Thomas DL. Genetic epidemiology of hepatitis C virus throughout Egypt. J Infect Dis. 2000;182(3):698-707.

16. Wantuck JM, Ahmed A, Nguyen MH. Review article: the epidemiology and therapy of chronic hepatitis C genotypes 4,5 and 6 . Aliment Pharmacol Ther. 2014;39(2):137-147.

17. Razavi H, Waked I, Sarrazin C, et al. The present and future disease burden of hepatitis $\mathrm{C}$ virus (HCV) infection with today's treatment paradigm. J Viral Hepat. 2014;21(Suppl 1):34-59.

18. Smith BD, Morgan RL, Beckett GA, et al; Centers for Disease Control and Prevention. Recommendations for the identification of chronic hepatitis C virus infection among persons born during 1945-1965. MMWR Recomm Rep. 2012;61(43):886.

19. Moyer VA; U.S. Preventive Services Task Force. Screening for hepatitis $\mathrm{C}$ virus in adults. US Preventive Services Task Force Recommendations Statement. Ann Intern Med. 2013;159(5):349-357.

20. Colin C, Lanoir D, Touzet S, Meyaud-Kraemer L, Bailly F, Trepo C; HEPATITIS Group. Sensitivity and specificity of third generation hepatitis $\mathrm{C}$ antibody detection assays: an analysis of the literature. $J$ Viral Hepat. 2001;8(2):87-95.
21. Chevaliez S, Pawlotsky JM. Hepatitis $\mathrm{C}$ virus serologic and virologic tests and clinical diagnosis of HCV-related liver disease. Int J Med Sci. 2006;3(2):35-40.

22. Joshi SN. Hepatitis C screening. Ochsner J. 2014;14(4):664-668.

23. Waked I, Doss W, El-Sayed MH, et al. The current and future disease burden of chronic hepatitis C virus infection in Egypt. Arab J Gastroenterol. 2014;15(2):45-52.

24. Estes C, Abdel-Kareem M, Abdel-Razek W, et al. Economic burden of hepatitis $\mathrm{C}$ in Egypt: the future impact of highly effective therapies. Aliment Pharmacol Ther. 2015;42(6):696-706.

25. Chen YP, Zhang Q, Dai L, Liang XE, Peng J, Hou JL. Is transient elastography valuable for high-risk esophageal varices prediction in patients with hepatitis-B-related cirrhosis? J Gastroenterol Hepatol. 2012;27(3):533-539.

26. Tsochatzis EA, Gurusamy KS, Ntaoula S, Cholongitas E, Davidson BR, Burroughs AK. Elastography for the diagnosis of severity of fibrosis in chronic liver disease: a meta-analysis of diagnostic accuracy. $J$ Hepatol. 2011;54(4):650-659.

27. Al Knawy B, Shiffman M. Percutaneous liver biopsy in clinical practice. Liver Int. 2007;27(9):1166-1173.

28. Castera L, Vergniol J, Foucher J, et al. Prospective comparison of transient elastography, Fibrotest, APRI, and liver biopsy for the assessment of fibrosis in chronic hepatitis C. Gastroenterology. 2005;128(2):343-350.

29. Sasso M, Miette V, Sandrin L, Beaugrand M. The controlled attenuation parameter (CAP): a novel tool for the non-invasive evaluation of steatosis using Fibroscan. Clin Res Hepatol Gastroenterol. 2012;36(1):13-20.

30. Ryder SD, Irving WL, Jones DA, Neal KR, Underwood JC; Trent Hepatitis C Study Group. Progression of hepatic fibrosis in patients with hepatitis C: a prospective repeat liver biopsy study. Gut. 2004;53(3):451-455.

31. Pritchett S, Cardenas A, Manning D, Curry M, Afdhal NH. The optimal cut-off for predicting large esophageal varices using transient elastography is disease specific. J Viral Hepat. 2011;18(4):e75-e80.

32. Chekuri S, Nickerson J, Bichoupan K, et al. Liver stiffness decreases rapidly in response to successful hepatitis $\mathrm{C}$ treatment and then plateaus. PLoS One. 2016;11(7): 0159413.

33. ANRS CO13 HEPAVIH Cohort. Regression of liver stiffness after sustained hepatitis $\mathrm{C}$ virus (HCV) virological responses among HIV/ HCV-coinfected patients. AIDS. 2015;29(14):1821-1830.

34. Hézode C, Castéra L, Roudot-Thoraval F, et al. Liver stiffness diminishes with antiviral response in chronic hepatitis C. Aliment Pharmacol Ther. 2011;34(6):656-663.

35. Wang JH, Changchien CS, Hung CH, et al. Liver stiffness decrease after effective antiviral therapy in patients with chronic hepatitis $\mathrm{C}$ : longitudinal study using FibroScan. J Gastroenterol Hepatol. 2010;25(5):964-969.

36. D'Ambrosio R, Aghemo A, Rumi MG, et al. A morphometric and immunohistochemical study to assess the benefit of a sustained virological response in hepatitis $\mathrm{C}$ virus patients with cirrhosis. Hepatology. 2012;56(2):532-543.

37. Balart LA, Lisker-Melman M, Hamzeh FM, Kwok A, Lentz E, Rodriguez-Torres M; LATINO study investigators. Peginterferon alpha2a plus ribavirin in Latino and Non-Latino Whites with HCV genotype 1: histologic outcomes and tolerability from the LATINO study. Am J Gastroenterol. 2010;105(10):2177-2185.

38. Mallet V, Gilgenkrantz H, Serpaggi J, Verkarre V, Vallet-Pichard A, Fontaine H, Pol S. Brief communication: the relationship of regression of cirrhosis to outcome in chronic hepatitis C. Ann Intern Med. 2008;149(6):399-403.

39. Maylin S, Martinot-Peignoux M, Moucari R, et al. Eradication of hepatitis $\mathrm{C}$ virus in patients successfully treated for chronic hepatitis C. Gastroenterology. 2008;135(3):821-829.

40. Poynard T, McHutchison J, Manns M, et al. Impact of pegylated interferon alfa- $2 \mathrm{~b}$ and ribavirin on liver fibrosis in patients with chronic hepatitis C. Gastroenterology. 2002;122(5):1303-1313.

41. Castera L. Noninvasive methods to assess liver disease in patients with hepatitis B or C. Gastroenterology. 2012;142(6):1293-1302.

42. Castera L, Foucher J, Bernard PH, et al. Pitfalls of liver stiffness measurement: a 5-year prospective study of 13,369 examinations. Hepatology. 2010;51(3):828-835. 
43. Wong GL, Wong VW, Chim AM, et al. Factors associated with unreliable liver stiffness measurement and its failure with transient elastography in the Chinese population. J Gastroenterol Hepatol. 2011;26(2):300-305.

44. Bonekamp S, Kamel I, Solga S, Clark J. Can imaging modalities diagnose and stage hepatic fibrosis and cirrhosis accurately? $J$ Hepatol. 2009;50(1):17-35.
45. Venkatesh SK, Yin M, Ehman RL. Magnetic resonance elastography of liver: technique, analysis, and clinical applications. J Magn Reson Imaging. 2013;37(3):544-555.

46. Venkatesh SK, Ehman RL. Magnetic resonance elastography of liver. J Magn Reson Imaging Clin N Am. 2014;22(3):433-446.
Hepatic Medicine: Evidence and Research

\section{Publish your work in this journal}

Hepatic Medicine: Evidence and Research is an international, peerreviewed, open access journal covering all aspects of adult and pediatric hepatology in the clinic and laboratory including the following topics: Pathology, pathophysiology of hepatic disease; Investigation and treatment of hepatic disease; Pharmacology of drugs used for the treatment

\section{Dovepress}

of hepatic disease. Issues of patient safety and quality of care will also be considered. The manuscript management system is completely online and includes a very quick and fair peer-review system, which is all easy to use. Visit http://www.dovepress.com/testimonials.php to read real quotes from published authors.

Submit your manuscript here: https://www.dovepress.com/hepatic-medicine-evidence-and-research-journal 